\title{
Forward Physics with the CMS Experiment at LHC
}

\author{
D. Sunar $\operatorname{Cerci}^{1, a, b}$
}

${ }^{1}$ Adiyaman University, Faculty of Arts and Sciences, Department of Physics, 02040 Adiyaman, Turkey.

\begin{abstract}
Forward physics measurements with the Compact Muon Solenoid (CMS) experiment, one of the two large multi-purpose experiments at the Large Hadron Collider (LHC) at CERN, cover a wide range of physics subjects. The forward calorimeters of CMS, HF and CASTOR, are used to collect data up to a pseudo-rapidity of 6.6. These detectors provide sensitivity to a large part of the total inelastic cross section, including diffractive events that produce particles only at forward rapidity, with the exception of very low mass diffraction. The results obtained with a centre-of-mass energy of $13 \mathrm{TeV}$ are presented. The measurements are compared to model predictions and provide valuable input for tuning of Monte Carlo models used to describe high-energy hadronic interactions.
\end{abstract}

\section{Introduction}

The LHC at CERN has started to deliver proton-proton collisions at the highest energy of $\sqrt{s}=13 \mathrm{TeV}$ ever reached by any other accelerator since 2015. Therefore, the CMS detector [1] at the LHC is a suitable machine which provides the opportunity to study forward physics. The forward physics programme at CMS mainly focuses on the physics that can be reached with the forward detectors HF and CASTOR at $3.0<|\eta|<5.2$ and $-6.6<\eta<-5.2$, respectively. Measurements in the forward region can be used to improve the knowledge of the content of the proton at small values of the momentum fraction $x$ carried by the partons and to provide inputs for parton density functions (PDFs). The hard scattering events are produced by the strong interactions of partons in a region where non-perturbative QCD and multiple partonic interactions (MPI) play an important role. Measurements performed in the forward region can shed light on MPI and the underlying events (UE) as well as provide useful information on the tuning of Monte Carlo (MC) event generators available.

\section{Measurements}

In the following the measurements performed with the data taken at a centre-of-mass energy $\sqrt{s}=13 \mathrm{TeV}$ in 2015 within the forward physics program of CMS are presented.

\footnotetext{
ae-mail: deniz.sunar.cerci@cern.ch

${ }^{\mathrm{b}}$ On behalf of the CMS Collaboration
} 


\subsection{Inelastic pp cross section}

One of the fundamental observables in high energy particle physics is the hadronic total cross section which has been measured in many experiments, covering several orders of magnitude in centre-ofmass energy $\sqrt{s}$. The hadronic cross section can be divided into two parts as elastic and inelastic interactions. In inelastic interactions, which can be described by non-perturbative and phenomenological models, either one or both protons dissociate as a result of colorless (diffractive) or colored (non-diffractive) exchange. Precise measurements of the hadronic cross sections provide valuable input for the phenomenological models as well as for the tuning of MC event generators.

The inelastic proton-proton cross section measurement at a centre-of-mass energy of $\sqrt{s}=13 \mathrm{TeV}$ is performed with the CMS detector, particularly with information from forward calorimeters, HF and CASTOR, at pseudorapidities of $3.0<\eta<5.2$ and $-6.6<\eta<-3.0$ [2]. Fig. 1 shows the comparison of corrected cross sections in various phase space regions to different models (left) and the same cross sections normalized to the cross section obtained for $\xi>10^{-6}$ (right). A visible cross section of $\sigma\left(\xi_{X}>10^{-7}\right.$ or $\left.\xi_{Y}>10^{-6}\right)=66.9 \pm 0.4$ (exp.) \pm 2.0 (lum.) mb is measured for $M_{X}>4.1 \mathrm{GeV}$ and $M_{Y}>13 \mathrm{GeV}$ where $M_{X}$ and $M_{Y}$ are the invariant masses of the two hadronic systems separated by the largest rapidity gap in the event. The visible cross section is extrapolated to the full inelastic phase space domain and is obtained as $71.3 \pm 0.5$ (exp.) \pm 2.1 (lum.) \pm 2.7 (ext.) mb. The increase from $\xi>10^{-6}$ to $\xi_{X}>10^{-7}$ or $\xi_{Y}>10^{-6}$ observed in data is reasonably described by most of the models used in the analysis. However, the hadronic scattering models predict higher cross section than the measured one.
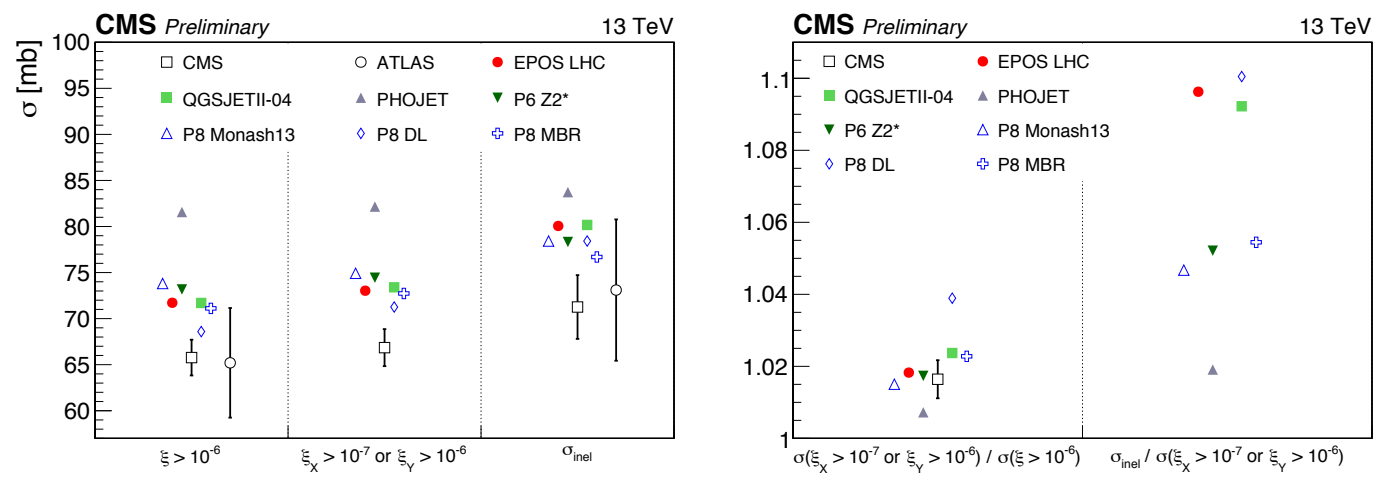

Figure 1. Left: Comparison of corrected cross sections in various phase space regions to different models. Right: the same cross sections scaled to the cross section obtained for $\xi>10^{-6}$ [2].

\subsection{Forward energy flow and transverse energy density}

The forward energy flow measurement is sensitive to the Underlying Event activity (UE) and parton shower dynamics. Therefore the measurement provides an opportunity to study multiple parton interactions (MPI), which are at the origin of UE activity. On the other hand, such a measurement can provide useful input for the tuning of existing hadronic interaction models.

The energy flow and transverse energy measurement [4] is performed with low luminosity $p p$ data at $\sqrt{s}=13 \mathrm{TeV}$ collected in 2015 in the pseudorapidity range $3.15<|\eta|<6.6$. The forward energy 
flow is defined as the total energy measured by the HF and CASTOR calorimeters by using the sum of all energy deposits of calorimeter towers above noise threshold $(5 \mathrm{GeV})$. The forward energy flow measurements presented here are obtained for two different event selections: soft inclusive inelastic events (INEL) allowing significant fraction of diffractive events and non-single diffractive enhanced events (NSD-enhanced) where diffractive contributions are effectively suppressed.

In order to have a comparison with model predictions the energy flow measured in calorimeters is corrected to the particle level by using GEANT4 [3] based MC detector simulation. The correction factors are defined as ratios of MC predictions at particle level and detector level where the detector level MC is defined as a fully simulated and reconstructed sample which goes through the same analysis chain as data. The average value of these correction factors is taken for the final correction. For INEL events, the event selection at the particle level is defined using the kinematic variable $\xi=M^{2} / s>10^{-6}$, which is the scaled mass of the largest (diffractively dissociated) system separated from the rest of the observed final state by the largest rapidity gap. NSD-enhanced event selection at particle level is done with a requirement of at least one particle except muon or neutrino on both sides with respect to the interaction point. The comparison of energy flow measurement as a function of $\eta$ for soft-inclusive-inelastic events to various MC models is shown in Fig. 2 (left). The ratio of data to MC models is shown in Fig. 2 (right). The gray band represents the total systematic uncertainties. Similar plots are also shown for non-single diffractive enhanced events in Fig. 3. In Fig. 4, the measured transverse energy density as a function of shifted pseudorapidity variable, $\eta^{\prime}=\eta-y_{\text {beam }}$, is compared to published CMS data at lower centre-of-mass energies and MC models. The results at different $\sqrt{s}$ are consistent in terms of $\eta-y_{\text {beam }}$, and the trends observed both in data and MC models support the limiting fragmentation hypothesis.
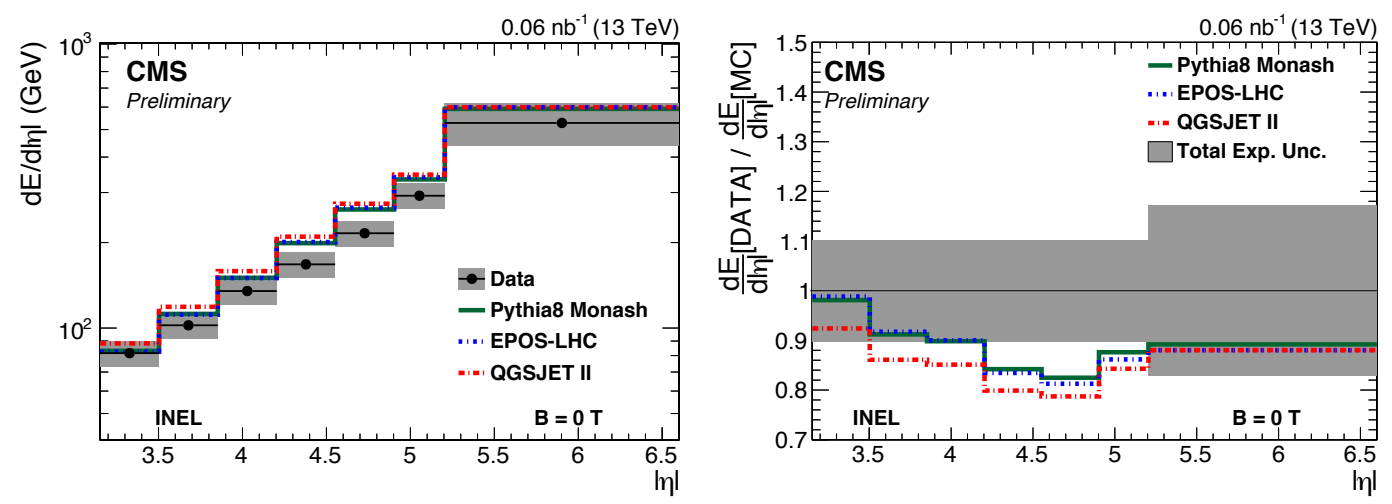

Figure 2. Left: Energy flow for soft-inclusive-inelastic events compared to various MC models: PYTHIA8, EPOS-LHC and QGSJET II. Right: Ratio of data to MC models [4].

\subsection{Pseudorapidity distributions of charged-particles}

A measurement of charged-particle pseudorapidity distributions, $\mathrm{d} N_{c h} / \mathrm{d} \eta$, produced in $p p$ collisions at $\sqrt{s}=13 \mathrm{TeV}$ is performed by CMS [6]. The charged-particles with a transverse momentum $p_{T}>0.5 \mathrm{GeV}$ in the pseudorapidity range $|\eta|<2.4$ are required. Four different event classes corresponding to different topologies of the final state particles and the activity measured in the forward region of the detector are selected. Pseudorapidity distributions of charged-particles for the inelasticenhanced, single diffractive enhanced and non-single diffractive enhanced event samples are shown 

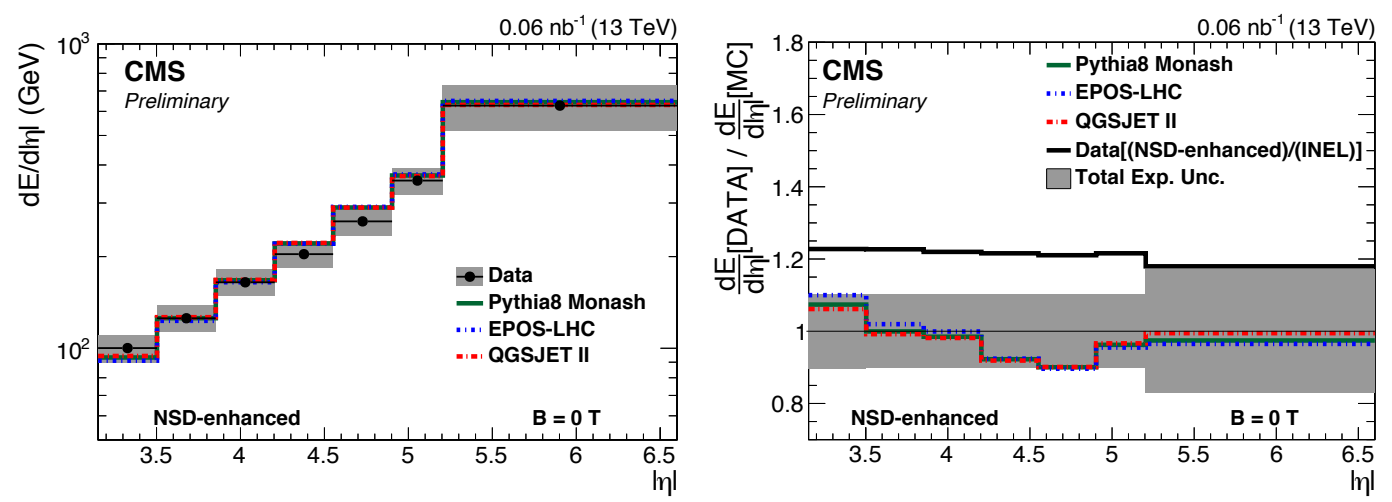

Figure 3. Left: Energy flow for non-single diffractive enhanced events compared to various MC models. Right: Ratio of data to MC models [4].

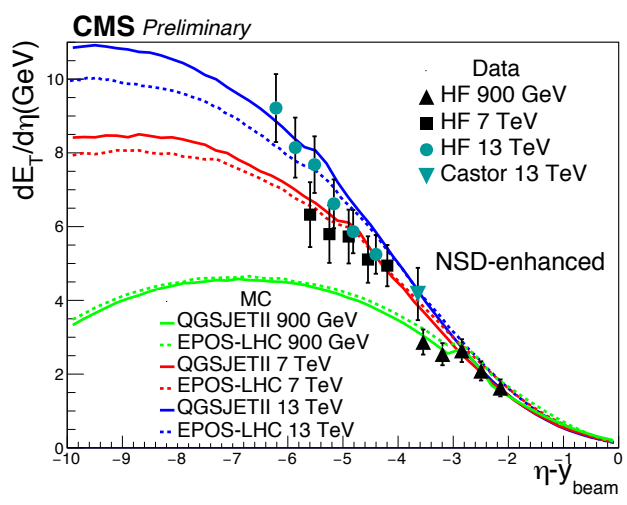

Figure 4. Comparison of transverse energy density measured by CMS at different centre-of-mass energies [5] and MC models [4].

in Fig. 5. The shaded bands around the data points represent the total systematic uncertainties. The measurements are compared to various $\mathrm{MC}$ event generators predictions and a reasonable agreement is observed between data and the MC predictions.

\subsection{Energy distribution in the very forward direction}

The energy spectrum, $\mathrm{d} N / \mathrm{d} E$, in the very forward direction $-6.6<\eta<-5.2$ is measured with the CMS detector in $p p$ collisions at $\sqrt{s}=13 \mathrm{TeV}$ [7]. The hadronic and electromagnetic energy spectra are also presented. The data corrected to the particle level is compared to models. A sensitivity is found on data to the production of neutral and charged mesons, which is an important input for the modeling of cosmic ray induced extensive air showers at ultra-high energies. The comparison of normalized total energy spectra in the pseudorapidity range $-6.6<\eta<-5.2$ to different $\mathrm{MC}$ and cosmic ray models is shown in Fig. 6. Despite the large systematic uncertainties where the energy 

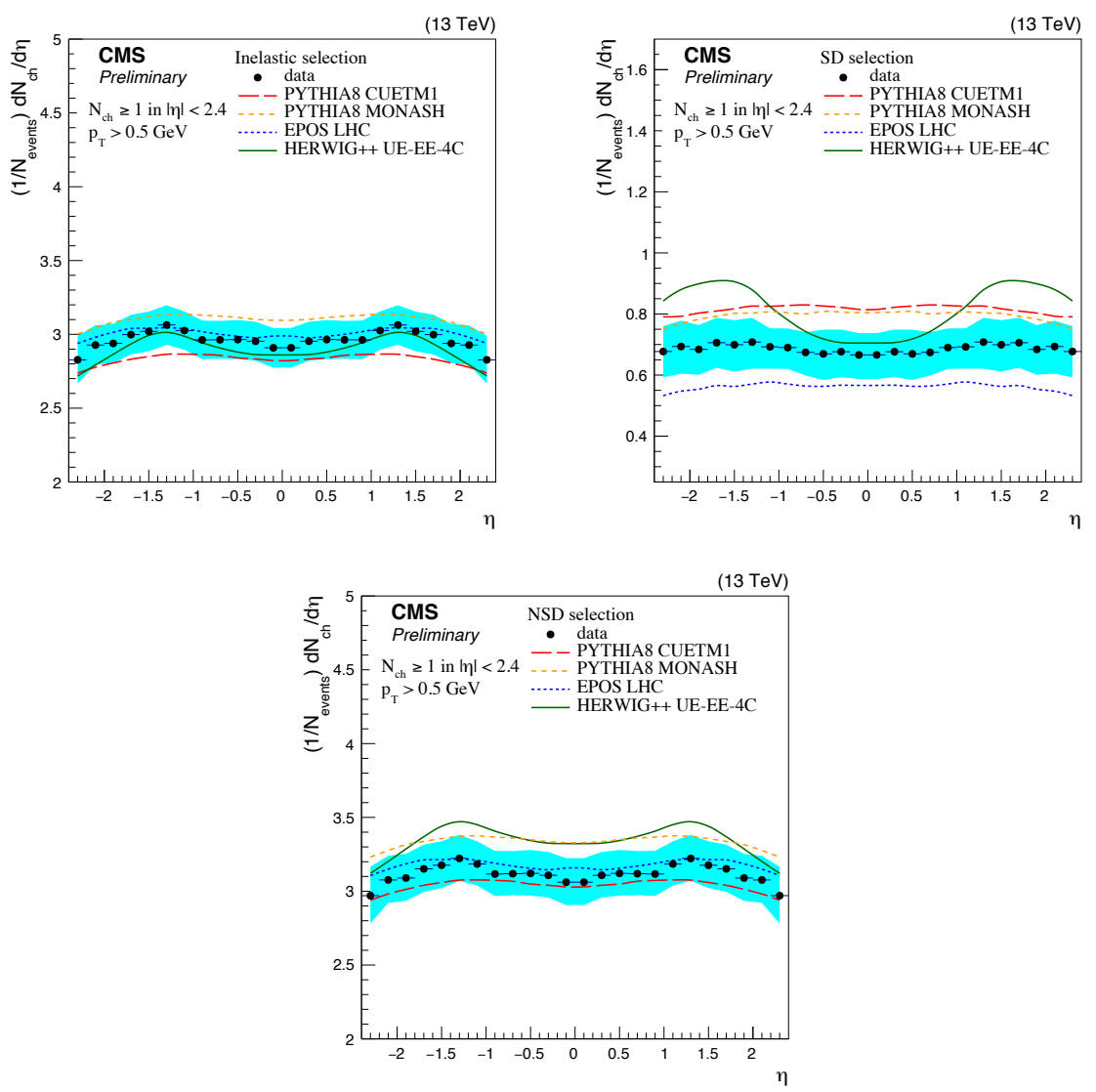

Figure 5. Pseudorapidity distributions of charged-particles for the inelastic-enhanced (top-left), single diffractive enhanced (top-right) and non-single diffractive enhanced (bottom) event samples [6].

scale is the dominant one, all models show significant differences with the data within the uncertainty band. The best agreement is observed between data and SIBYLL 2.1.

\subsection{Very forward inclusive jet cross section}

The very forward inclusive jet cross section is sensitive to radiation of partons, underlying partonic QCD processes and to the parton density functions (PDF) of the incoming hadrons. As shown by the previous measurements, jet cross sections can be described by perturbative QCD over several orders of magnitude in $Q^{2}$ and $x$, where $Q$ is the scale of scattering and $x$ is the longitudinal momentum fraction of the parton.

The very forward inclusive jet cross section measurement presented here is based on the very forward detector of CMS, CASTOR, and $p p$ collisions recorded in 2015 at $\sqrt{s}=13 \mathrm{TeV}$. Jets are reconstructed using the anti- $k_{t}$ algorithm [9] with a radius parameter of $R=\sqrt{\Delta \phi^{2}+\Delta \eta^{2}}=0.5$ and are required to have their axis between $-6.6<\eta<-5.2$. The transverse momentum of the jets must be in the range $3<p_{T}<13 \mathrm{GeV}$ and various jet quality criteria are applied to remove fake jets. The 

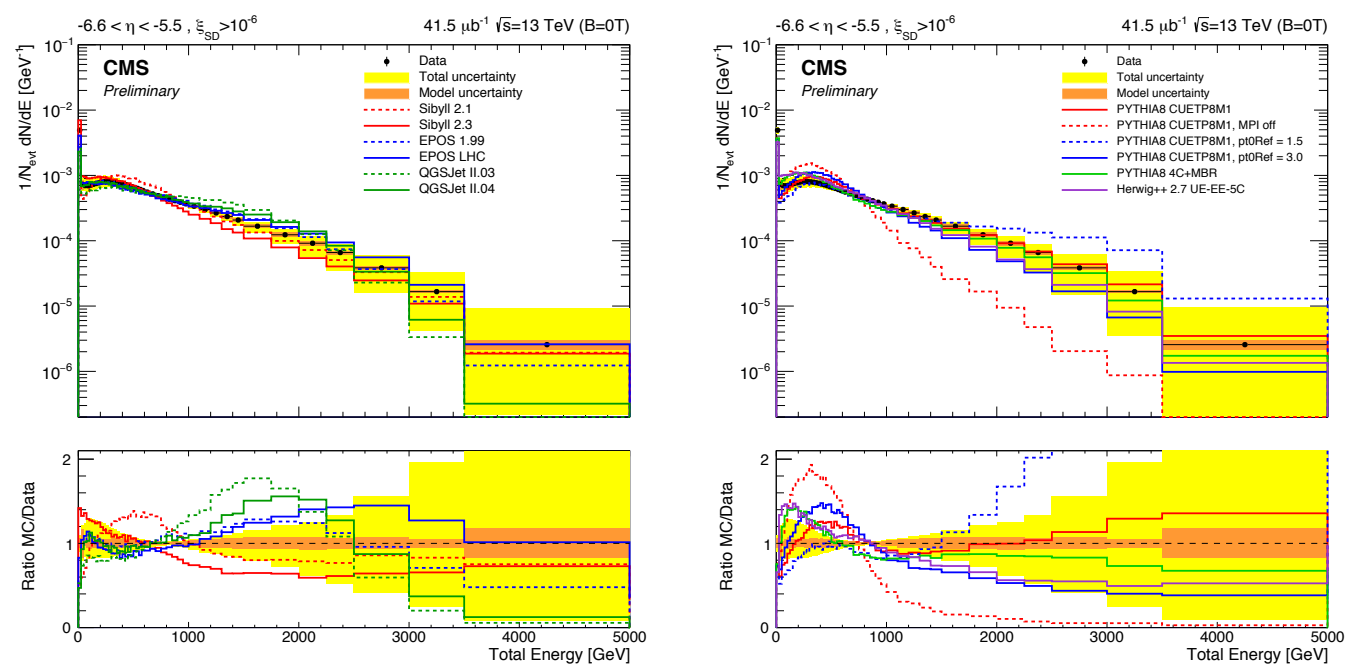

Figure 6. Comparison of normalized total energy spectrum to various MC models (left) and to PYTHIA8 tunes (right) [7].
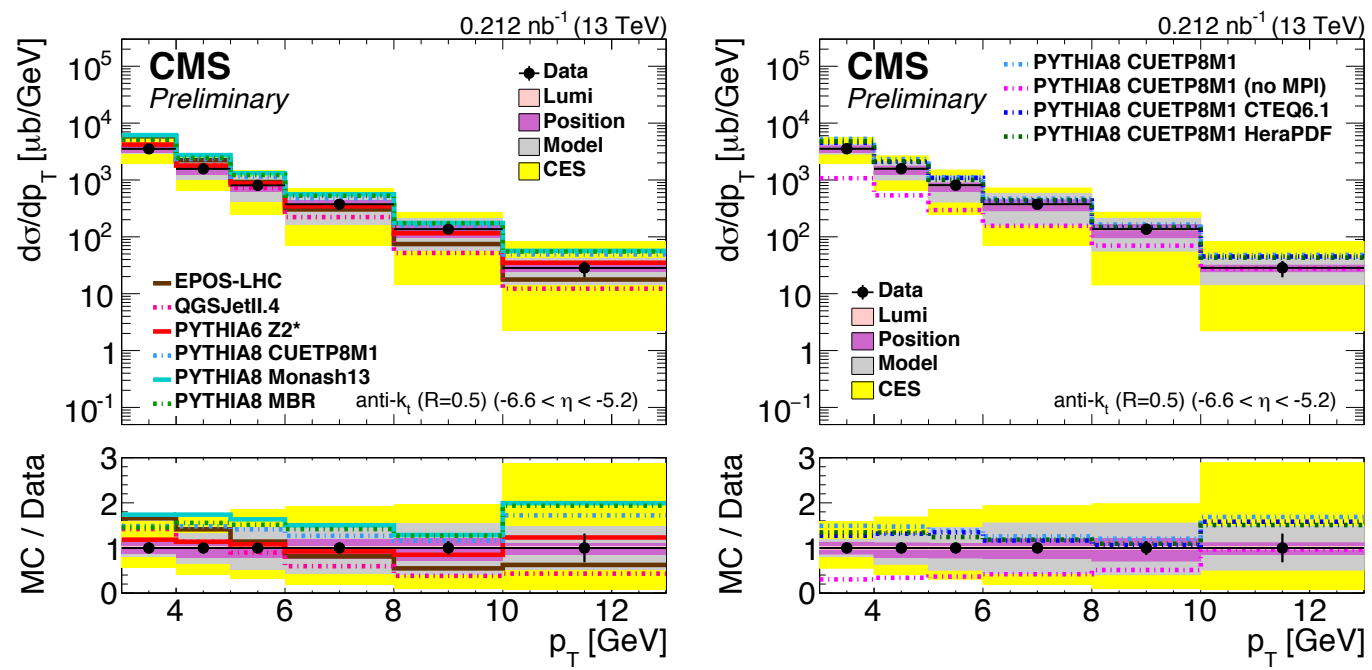

Figure 7. The measured very forward inclusive jet cross-section at $\sqrt{s}=13 \mathrm{TeV}$ compared to various MC models (left) and to PYTHIA8 tune CUETP8M1 with different PDFs (right) [8].

corrected cross section measurement of the very forward inclusive jet production is normalized by the luminosity and is shown in Fig. 7. The yellow band represents the total systematic uncertainty. The dominating systematic uncertainties on the very forward inclusive jet cross section measurement are the jet energy scale of CASTOR (CES), model dependence and the alignment of CASTOR. Within the current experimental uncertainty all models are consistent with the data. However, an overestimation 
is observed in all the PYTHIA tunes whereas the cosmic ray MC models EPOS-LHC and QGSJETII.4 underestimate the data. A high sensitivity to MPI is observed, but the measurement is found to have a moderate sensitivity to the underlying PDF set of the model.

\section{Summary}

An extensive list of forward physics measurements performed with data taken in 2015 at $\sqrt{s}=13 \mathrm{TeV}$ is presented. The measurements presented here can be used as input for tuning of existing hadronic interaction models. The collection of measurements in the forward region is an important baseline for more exclusive analyses and shows that forward physics is well understood within the systematic uncertainties at a $\sqrt{s}=13 \mathrm{TeV}$ scale collected by CMS.

\section{Acknowledgments}

The author would like to acknowledge the Adiyaman University Scientific Activity Foundation and the organizers of ICNFP2016 for the funding.

\section{References}

[1] S. Chatrchyan et al. [CMS Collaboration], JINST 3 S08004 (2008)

[2] CMS Collaboration, CMS Physics Analysis Summary CMS PAS FSQ-15-005, (2016)

[3] S. Agostinelli et al., Nuclear Instruments and Methods in Physics Research Section A: Accelerators, Spectrometers, Detectors and Associated Equipment 506, no. 3, 250 - 303, (2003)

[4] CMS Collaboration, CMS Physics Analysis Summary CMS PAS FSQ-15-006, (2016)

[5] S. Chatrchyan et al. [CMS Collaboration], JHEP 11 148, Erratum JHEP 02 (2012) 055, (2011)

[6] CMS Collaboration, CMS Physics Analysis Summary CMS PAS FSQ-15-008, (2016)

[7] CMS Collaboration, CMS Physics Analysis Summary CMS PAS FSQ-16-002, (2016)

[8] CMS Collaboration, CMS Physics Analysis Summary CMS PAS FSQ-16-003, (2016)

[9] M. Cacciari, G. P. Salam, and G. Soyez, JHEP 04 063, (2008) 